\title{
Facile and Low-Waste Self-Digitization of Samples by Oil-Triggered, Template-Confined Dewetting on a Networked Microwell Array
}

\author{
Nankun Xiong, ${ }^{\dagger}$ Anyan Wang, ${ }^{\ddagger}$ Tengbao Xie,${ }^{\dagger}$ Tianbao Hu, ${ }^{\dagger}$ Qiang Chen,,${ }^{\ddagger}$ Qiang Zhao,,${ }^{\dagger}$ and Gang \\ $\mathrm{Li}^{*}, \dagger$ \\ †Defense Key Disciplines Lab of Novel Micro-Nano Devices and System Technology, Key Laboratory of Optoelectronic \\ Technology and Systems, Ministry of Education, Chongqing University, Chongqing 400044, China \\ *Institute of Fluid Measurement and Simulation, China Jiliang University, Hangzhou 310018, China
}

\begin{abstract}
In this work, we present a simple, straightforward, and robust method for spontaneously digitizing samples into an array of discrete volumes. The method is based on an oil-triggered, template-confined dewetting phenomenon. To realize the dewetting-induced sample digitization, an aqueous sample is firstly infused into a networked microwell array (NMA) through a predegassing-based self-pumping mechanism, and then an immiscible oil phase is applied over the surface of NMA chip to induce the templated dewetting. Due to a periodic interfacial tension heterogeneity, such dewetting ruptures the sample at the thinnest parts (i.e., connection channels) and spontaneously splits the sample into droplets in individual microwells. Without requiring any complex pumping or valving systems, this method can discretize a sample into tens of thousands of addressable droplets in a matter of minutes with nearly $98 \%$ usage. To demonstrate the utility and universality of this self-digitization method, we exploited it to discretize samples into 40,233 wells for digital PCR assay, digital quantification of bacteria, and self-assembly of spherical colloidal photonic crystals. We believe this facile technique will be useful in a broad range of applications where partitioning of samples into a large number of small individual volumes is required.
\end{abstract}

Sample digitization is a crucial operation in many chemical and biological assays. ${ }^{1-4}$ By discretizing an initial sample into a large number of equal volume subunits, single target entities can be isolated from non-target species which enables new functionality for myriad applications including digital quantitation of nucleic acid or protein, ${ }^{5,6}$ single-cell analysis, ${ }^{7,8}$ protein crystallization, ${ }^{9,10}$ and drug discovery. ${ }^{11,12}$ Conventional approaches to sample discretization include nebulization and mechanically agitated emulsification. ${ }^{13,14}$ Although these methods can massively produce discretized droplets, they have limitations in the monodispersity and reproducibility of droplets. Moreover, it is difficult for these methods to continuously monitor individual droplets which is needed for studying the temporal processes of individual entities. Recently, a variety of microfluidic methods have been proposed for sample digitization. Among them, droplet microfluidics is the most popular approach to sample digitization, which produces a large number of monodisperse aqueous droplets in an oil phase using hydrodynamic interactions. ${ }^{15,16}$ Although droplet-based microfluidic digitization methods are powerful and fully-developed, they normally require complex and precise pumping equipment. To stabilize the water-oil interface and prevent droplet coalescence, these methods also require the addition of surfactants in the aqueous or oil phase, which may interfere with the reaction or assay. ${ }^{17}$ In addition, indexing is also a challenge for droplet microfluidic platforms since droplets are free to move throughout the experiment, which hinders time-lapse studies of large numbers of individual droplets. As an alternative to sample digitization, "static" microfluidic methods supporting the generation of arrays of physically isolated droplets have been developed. By confining droplets into static and spatially-defined arrays, these methods allow indexing and monitoring of droplets over time and also obviate the need for any surfactant addition. Typical "static" microfluidic digitization systems include pneumatically actuated valve arrays, ${ }^{18}$ SlipChip, ${ }^{19}$ OpenArray, ${ }^{20}$ trapping well arrays, ${ }^{21}$ and hydrophilic-in-hydrophobic patterns. ${ }^{22}$ Although these "static" microfluidic digitization systems offer the major advantage over the droplet-based approaches, most of them still require expensive external pumping equipment, accessory sample loading components, or complex microfabrication to achieve sample digitization. More recently, in order to lessen reliance on complex external pumping equipment and simplify the fluid operation for end-users, a degassing-driven self-pumping mechanism was recently introduced for "static" sample digitization. ${ }^{23-27}$ By employing the air absorption of the pre-degassed polydimethylsiloxane (PDMS) substrate to create pumping pressure, this power-free self-digitization technology has minimized the number of liquid control components, which is favorable to portable applications. In most cases, the power-free self-digitization devices consist of an array of microchambers, each row of microchambers connecting to a main microchannel. During sample digitization, such "microchamber array + main channel" features normally require an oil flushing step to remove the excess sample from the main channel after sample priming and thus isolate the aqueous sample in each microchamber. However, due to the rapid decay of pumping pressure created by the pre-degassed PDMS substrates and high viscosity of the oil phase, the oil flushing process is usually lengthy, which is unfavorable, if rapid analyses are required, e.g., pointof-care diagnostics. Furthermore, most existing methods require an excess of sample volume for sample digitization, making it unsuitable for analyzing very limited quantities of 
samples, e.g., a drop of blood from a needle prick. Therefore, it remains challenging for microfluidic platforms to achieve rapid, cost-effective, and low-sample-loss digitization. To address these challenges, we recently presented a rapid and facile method for spontaneous sample digitization, ${ }^{27}$ which uses a reversible channel-well assembly chip coupled with predegassing-based self-pumping mechanism to achieve an easy, fast, and large-scale sample partitioning. However, due to the detachable channel-well configuration, it requires tedious alignment and assembly which can hinder non-specialist users from using this sample digitization method.

In this work, we present a simpler and more straightforward method for spontaneously digitizing samples into tens of thousands of discrete volumes in less than one and a half minutes with about $98 \%$ usage. By combining the degassing-driven selfpumping mechanism and template-confined dewetting effect, this method spontaneously and rapidly discretizes an aqueous sample into a high-density networked microwell array without the need for any complex pumping/valving systems or tedious alignment steps. Unlike most (if not all) power-free methods for sample digitization, this method allows for automated, flowfree isolation of trapped sample in microwells without oil flushing operation, which further reduces the complexity of the fluid operation and is also favorable to minimal sample-loss during sample digitization. To demonstrate the utility and universality of this self-digitization method, we exploited it to discretize samples into 40,233 wells for digital PCR assay, digital quantification of bacteria, and self-assembly of spherical colloidal photonic crystals. We envision that our proposed method will provide a facile and versatile tool for a variety of digital assays or synthesis in the future.

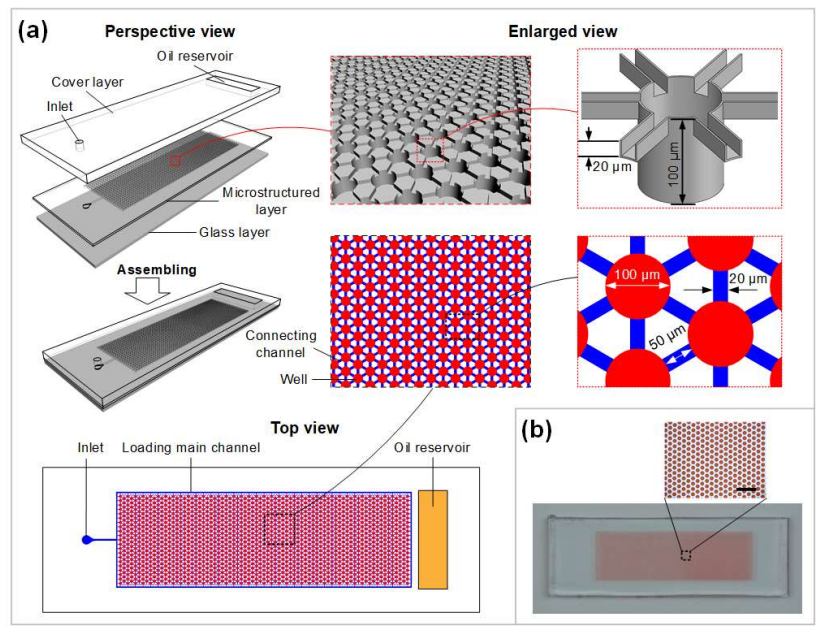

Figure 1. Design and components of the NMA chip used for dewetting-induced self-digitization. (a) Schematic of the NMA chip. The chip consists of three layers: a PDMS cover layer, a microstructured PDMS sheet, and a glass supporting layer. (b) Photograph of an NMA chip containing discretized samples, scale bar is $500 \mu \mathrm{m}$.

\section{RESULTS AND DISCUSSION}

Device Design and Operation. The basic design of a dewetting-induced self-digitization (SD) chip is depicted in Figure 1. The chip consists of a networked microwell array (NMA) and a rectangular reservoir. In the NMA, the microwells are arranged in a honeycomb manner, where inner individual microwells are connected to the surrounding six neighboring microwells while peripheral microwells connected to the main channel by narrow and shallow microchannels. Such a networked feature allows one sample loading to fill all the microwells with minimal sample amounts and time. Furthermore, its periodic surface heterogeneity can be used to guide the dewetting of the impregnated aqueous sample in an ordered fashion, leading to spontaneous sample digitization. Figure 2 shows the operation procedure of the dewettinginduced self-digitization chip for sample digitization (also see Movie S1). First, the inlet of the chip was sealed with tape, and then the sealed chip was placed in a vacuum condition $(\sim 10$ $\mathrm{kPa}$ ) for $1 \mathrm{~h}$ to build up a negative pressure in the closed microchannel/ microwell system for self-priming. ${ }^{27}$ After removal of the chip from the vacuum, a pipette tip holding aqueous sample $(\sim 35 \mu \mathrm{L})$ was immediately inserted through the tape and into the inlet of the chip. Driven by the negative pressure created in the chip during the degassing process, the sample solution was automatically sucked into the microchannels and quickly filled all the available space in the closed system including microchannels and microwells. ${ }^{23-29}$ Subsequently, a few drops of oil having high wettability to PDMS (e.g., silicone oil or hexadecane) were added to the oil reservoir. Once the sample filled all of the channels and microwells, the cover layer was peeled off from the microstructured layer. Due to its high wettability to PDMS, the oil leaked from the oil reservoir spontaneously spreads over the surface of the microstructure layer to trigger the dewetting of the impregnated aqueous sample at narrow well-to-well channels, leading to the isolation of sample in individual microwells and thus sample discretization. In contrast to traditional discontinuous dewetting which creates liquid arrays by draining or scraping the excess liquid from a molded or patterned surface, ${ }^{30,31}$ this method does not require any delicate handling of liquid or devices (e.g., carefully controlling the draining or scraping speed), thus providing a simple and robust route to sample digitization. More importantly, the primed sample can be transformed into discretized droplets in the NMA chip with nearly zero waste (only wasting a tiny sample loaded in the main channel). For our NMA chip, the volume of the main channel is about $2 \%$ of the total volume of channel-microwell space. Thus, it can be inferred that the usage ratio of sample is about 98\% (refer to Section S1 in Supporting Information for more details), which is favorable for analyzing very limited or precious samples.

Mechanism of Self-Digitization Induced by Oil-Triggered, Template-Confined Dewetting. Dewetting of liquid on solid surfaces is a spontaneous physical phenomenon driven by the total interfacial energy minimization, which induces the rupture of large continuous liquid and the formation of small droplets. The idea of templated dewetting is to use a pre-patterned or structured substrate to control liquid breakup at specific sites; ${ }^{32}$ that is, dewetting is driven by a physically and chemically defined substrate surface topography rather than by random intrinsic inhomogeneities, thus forming ordered liquid patterns. The most critical factor of determining the spatially-ordered dewetting is the topography features of the templates. In the case of our study, a networked microwell array serves as a template for inducing dewetting in an ordered manner to form defined arrays of droplets. Unlike solid-state dewetting which is generally activated by thermal treatment, ${ }^{32}$ the dewetting of liquid in the networked microwell array is triggered by the application of an oil phase, which not only minimizes sample evaporation but also is more compatible with biological assays 
than thermal treatment. As shown in Figure 3a, before peel-off of the cover layer, the top of the impregnated aqueous sample is nearly flat. Once the removal of the cover layer makes the oil phase cover the aqueous sample, to minimize the interfacial energy, the shape of the top of the aqueous liquid becomes convex. Due to different geometrical dimensions, the curvatures of the water/oil interfaces at the microwell and connection microchannel are notably distinct. Generally, the interface curvature is specified by the radii of curvature along two orthogonal directions, say $R_{1}$ and $R_{2}$. At the connection microchannel, the radii of curvature are:

$$
R_{1}=\frac{w}{|2 \cos \theta|}
$$

and

$$
R_{2} \sim \infty
$$

where $R_{1}$ and $R_{2}$ are the curvature radius of water/oil interface along the direction vertical to and parallel to the connection microchannel, respectively; $w$ is the width of the connection microchannel and $\theta$ is the contact angle between the aqueous liquid surface and the solid (i.e., the microchannel wall). According to the Young-Laplace equation, the pressure of the aqueous liquid at the connection microchannel is given as

$$
P_{\text {channel }}=P_{\mathrm{atm}}+\gamma_{\mathrm{w} / \mathrm{o}}\left(\frac{1}{R_{1}}+\frac{1}{R_{2}}\right)=P_{\mathrm{atm}}+\gamma_{\mathrm{w} / \mathrm{o}} \cdot \frac{|2 \cos \theta|}{w}
$$

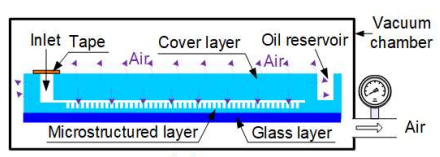

(a)
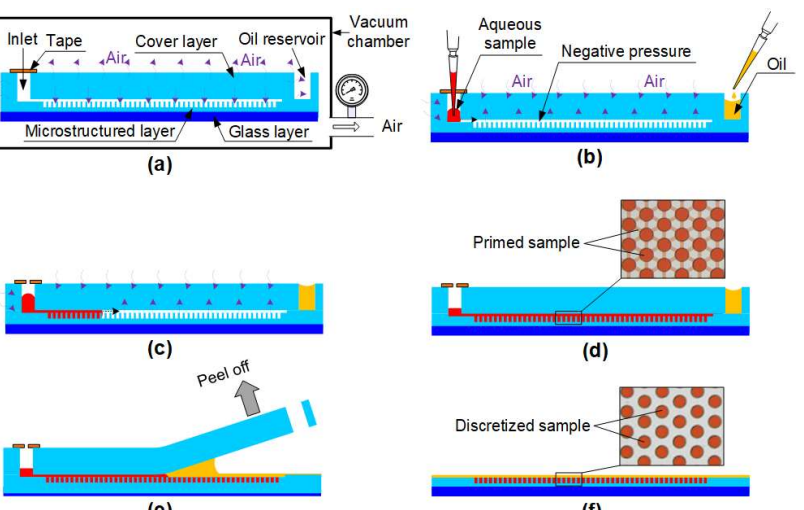

(e)

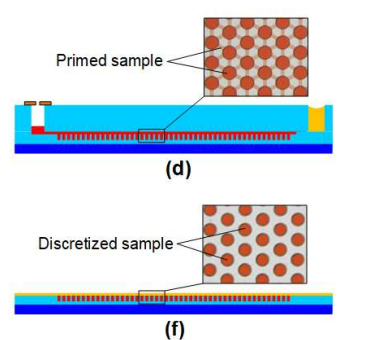

Figure 2. Schematic illustration of the operation procedure of the NMA chip for sample digitization. (a) degassing of the chip in a vacuum chamber; (b) removing the chip from vacuum conditions, adding an aliquot of sample in the inlet and a few drops of oil in the oil reservoir; (c) aspiration of the sample into the microchannels under the negative pressure created by the degassed PDMS substrate; (d) sample priming of all channels and wells; (e) peeling off the cover layer from the microstructured layer; (f) discretization of the primed sample into all wells induced by oil-triggered dewetting.

where $P_{\text {atm }}$ is the atmospheric pressure and $\gamma_{\mathrm{w} / \mathrm{o}}$ is the interfacial surface tension. Similarly, at the microwell, the radii of curvature are:

$$
R^{\prime}{ }_{1}=R_{2}^{\prime}=\frac{d}{|2 \cos \theta|}
$$

where $R_{1}{ }^{\prime}$ and $R_{2}{ }^{\prime}$ are the curvature radius of water/oil interface at the microwell along the direction vertical to and parallel to the connection microchannel, respectively; $d$ is the diameter of the microwell. The pressure of the aqueous liquid at the microwell is given as

$$
P_{\text {well }}=P_{\text {atm }}+\gamma_{\mathrm{w} / \mathrm{o}}\left(\frac{1}{{R^{\prime}}_{1}}+\frac{1}{{R^{\prime}}_{2}}\right)=P_{\mathrm{atm}}+\gamma_{\mathrm{w} / \mathrm{o}} \cdot \frac{|4 \cos \theta|}{d}
$$

Thus, there is a pressure difference between aqueous liquids at the microwell and those at the connection microchannel, which is calculated as

$$
\Delta P=P_{\text {channel }}-P_{\text {well }}=\gamma_{\mathrm{w} / \mathrm{o}} \cdot|2 \cos \theta| \cdot\left(\frac{1}{w}-\frac{2}{d}\right)
$$

As shown in Figure 1a, $w$ is $20 \mu \mathrm{m}$ and $d$ is $100 \mu \mathrm{m}$, so that $\Delta P>$ 0 . Such pressure difference causes the aqueous liquid to flow toward the microwell (low pressure region) from the connection microchannel (high pressure region), and thus the aqueous liquid in the microchannel becomes thinner and finally ruptures, achieving sample digitization.

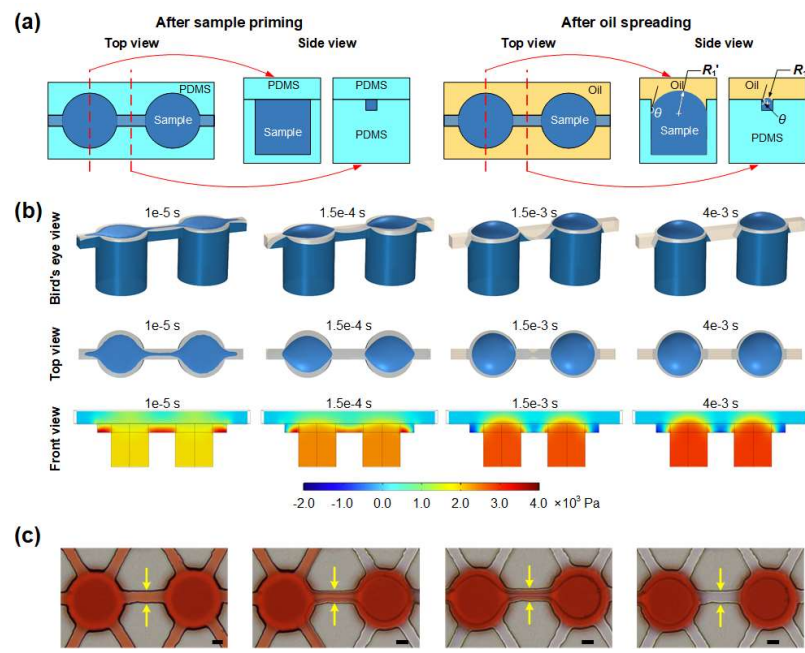

Figure 3. Description and modeling of oil-triggered, templated dewetting in a networked microwell array chip. (a) Schematic of the oil-triggered, templated dewetting mechanism. The Laplace pressure gradient resulted from the spatial curvature difference drives the aqueous liquid in the microchannel toward the microwell, causing the thinning and splitting of the liquid ligament in the microchannel. (b) Sequential simulation images showing the selfsplitting of the aqueous sample in a connection channel induced by oil-triggered dewetting. (c) Sequential bright-field microscope images showing the pinch-off of the aqueous sample in a connection channel during the oil-triggered dewetting process. Scale bar represents $20 \mu \mathrm{m}$.

To gain a better insight into the liquid dynamics during sample digitization, we conducted a numerical simulation of the oil-triggered, template-confined dewetting using the finite element method (COMSOL Multiphysics 5.6). The dewettinginduced self-digitization can be roughly divided into two stages: i) convex interface formation process in which the oil phase is introduced to displace the solid PDMS during peeling off and cover the aqueous liquid in the networked microwell array, and then the minimization of overall surface energies deforms the water/oil interfaces to form convex shapes; and ii) Laplace pressure-driven flow process in which the Laplace pressure induced by the convex oil/water interfaces provides the driving force for thinning and rupture of the aqueous liquid at the connection microchannels. In the numerical simulation, to simplify the model calculation, we directly applied the oil phase over the chip surface at $t=0 \mathrm{~s}$. Figure 3b shows the simulated local dewetting process in the NMA chip. Once the oil phase is applied, the water/oil interfaces form convex shapes (see Movie S2). It is clear that the water/oil interface at the connection 
microchannel has a larger curvature while the water/oil interface at the microwell has a smaller curvature (Figure 3a and Movie S3). Such curvature difference generates an internal Laplace pressure gradient which pushes the aqueous liquid away from the connection microchannel towards the neighboring microwells. Thus, the aqueous liquid becomes thinner and forms a neck at the center of the connection microchannels as a result of local mass reduction. The formation of this neck further increases the Laplace pressure gradient due to the increase in curvature by the reduction of local liquid ligament radius, and thus accelerating the liquid ligament thinning and leading to its eventual pinch-off and a local dewetting of aqueous liquid at the connection microchannel (see Movie S4). By exploiting this local dewetting effect, the NMA chip isolates the aqueous liquid into microwells, achieving self-digitization. Figure $3 \mathrm{c}$ shows a series of frames obtained from the video recording the oil-triggered, template-confined dewetting process. These images illustrate the qualitative features of the oil-triggered, templateconfined dewetting process and agree well with those predicted by the numerical simulation.

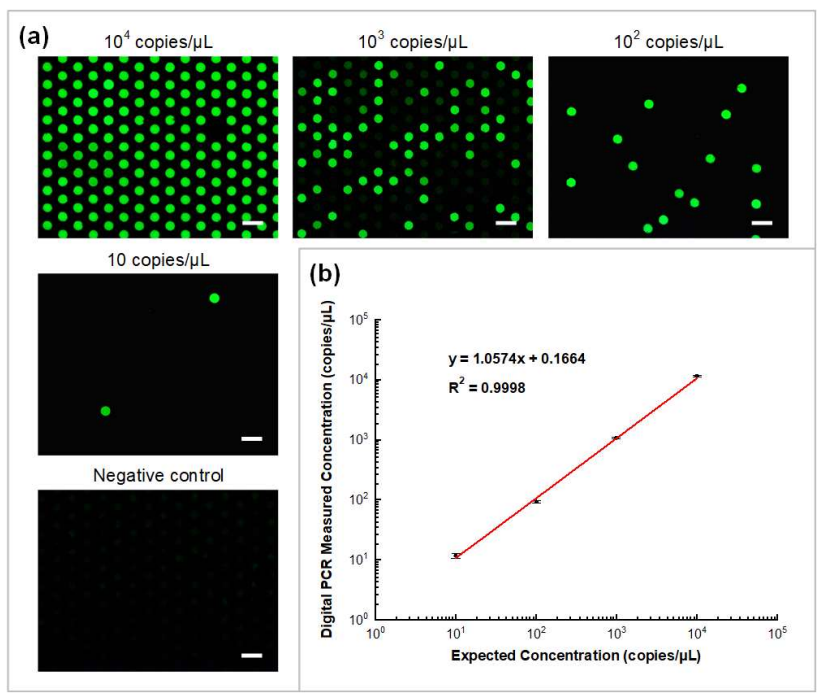

Figure 4. Digital PCR on the NMA chip: (a) Fluorescence images showing the representative digital PCR results with a serial dilution of target DNA template ranging from 10 copies $/ \mu \mathrm{L}$ to $10^{4}$ copies $/ \mu \mathrm{L}$. The negative assay was in control when no target template was loaded. Scale bar represents $200 \mu \mathrm{m}$. (b) A log plot showing the linear relationship between the measured value (copies $/ \mu \mathrm{L})$ in the NMA chip and the expected copy number per reaction (Note: each data point represents the mean \pm standard deviation of three independent experiments). The measure values matched well with the expected values according to Poisson statistics $\left(\mathrm{R}^{2}=0.9998\right)$.

Digital PCR. To explore the capability of our selfdigitization method, we firstly applied it to a digital PCR test for detecting KRAS G12A mutation. KRAS G12A is one of the most common KRAS-activating mutations, occurring in many cancer types, such as lung, colon, colorectal, pancreatic, and ovarian tumors. ${ }^{33}$ For digital PCR detection of KRAS G12A, a serial dilution of DNA template solutions (ranging from 10 copies $/ \mu \mathrm{L}$ to $10^{4}$ copies $/ \mu \mathrm{L}$ ) was prepared. After premixing, four PCR mixtures obtained from the serially diluted DNA template solutions and a negative control (not including template) were introduced into five pre-degassed NMA chips, respectively, and partitioned into the whole array of microwells with the oil-triggered dewetting. After partitioning, each NMA chip was sealed by a glass slide coated with a thin layer of uncured PDMS to form a glass-PDMS-glass sandwich configuration, which can minimize the evaporation of liquid in the chip during thermo-cycling. Subsequently, the assembled chips were placed in a thermal cycler with a flat block (Mastercycler nexus flat, Eppendorf, German) to perform PCR thermocycling. After PCR, the chips were transferred to a fluorescence microscope for imaging. Figure $4 \mathrm{a}$ shows representative fluorescence images obtained from different concentrations of DNA template. It can be found that the numbers of positive partitions increased with the concentrations of the DNA template. Furthermore, no positive signals were observed in the chip corresponding to the negative control reaction, suggesting that no contamination or unspecific amplification occurred. For quantitative analysis of the results, the obtained fluorescence images were analyzed by counting the negative and positive partitions with ImageJ software (http://rsbweb.nih.gov). Finally, the average copy number of DNA template initially present in each chip was calculated by using Poisson statistics. By fitting the measured data to the expected template concentration values, a good linear correlation $\left(\mathrm{R}^{2}=0.9998\right)$ was observed (Figure $\left.4 \mathrm{~b}\right)$ and the slope and intercept of the linear correlation were determined to be 1.0021 and 0.2964 , respectively. These values demonstrate the capability of our self-digitization method to absolutely quantify DNA. Note that our NMA chip was able to detect template DNA at concentrations as low as 10 copies $/ \mu \mathrm{L}$, with a dynamic range of over 4 orders of magnitude, demonstrating that the NMA chip is a useful platform for highly sensitive and precise genetic analysis.

Digital Quantification of Viable Bacteria. Precise counting of viable bacteria is important to a variety of microbiological applications such as clinical diagnosis, food quality monitoring, and antibiotic susceptibility testing. To further test the quantitative performance of our self-digitization method, we applied it to digital quantification of bacteria. This quantification method for the detection of bacteria is similar to digital PCR in quantitative strategy but plays the same role as the colony counting and thus can be named as "digital CFU". In the same way, a sample is stochastically partitioned into a large number of microwells with the oil-triggered dewetting selfdigitization method, allowing most microwells to be loaded with single or no bacteria. After several hours of incubation, enumeration can be carried out on micro-colonies proliferated from single bacteria to quantify the initial bacterial concentration in the sample. To facilitate the observation of "microcolonies" proliferated from single bacteria, a resazurinamplified fluorescence detection was adopted. ${ }^{34}$ Resazurin is a fluorescent dye used as a cell viability indicator, which can be reduced by intracellular electron receptors (e.g., NADH and FADH) into strongly fluorescent molecule resofurin. Therefore, in this case, the fluorescence signal in a microwell reflects the presence of viable and proliferating bacteria. This strategy not only accelerates the enumeration of microcolonies proliferated from individual bacteria but also allows enumeration of individual microcolonies using simple digital imaging setups. Here, we use AlamarBlue (a resazurin-based dye) to achieve resazurin-amplified fluorescence detection of bacteria in the NMA chip. Prior to each experiment, samples were prepared by mixing broth, AlamarBlue, and E. coli JM109 at predetermined concentrations. The prepared samples were discretized into microwells by the oil-triggered, template-confined dewetting approach and then the devices were incubated at $37{ }^{\circ} \mathrm{C}$ in an 
incubator to promote bacterial proliferation within microwells. After 4 hours of incubation, all chips were examined by fluorescent microscopy to count the positive microwells containing microcolonies in each chip. Figure 5a shows representative fluorescence images of the NMA chips loaded with different concentrations of $E$. coli JM109 after incubation. Furthermore, we also compared the quantitative results (i.e. calculated number of CFUs in the samples) obtained with our digital CFU assay and those from the conventional plate counting method. As shown in Figure 5b, the $E$. coli concentrations measured with the NMA chip-based digital CFU correspond very well to those measured with the conventional plate counting method $\left(\mathrm{R}^{2}=0.9992\right)$ in the entire 4 log range of the measured sample concentrations, demonstrating the capability of the NMA chip-based digital CFU method to quantify microbes. Importantly, the NMA chip-based digital CFU method offers four distinct advantages over the conventional plate counting method: i) faster bacteria detection; ii) lower sample/reagent consumptions; iii) less labor-consuming; iv) wider dynamic range of concentrations. Although the previously developed droplet digital CFU methods possess the same advantages, the requirement of complex and precise pumping equipment limits theis applications. Due to its simplicity, rapidity, and precision in the quantification of viable bacteria, this NMA chip-based method provides an extremely useful tool for various microbiological applications.

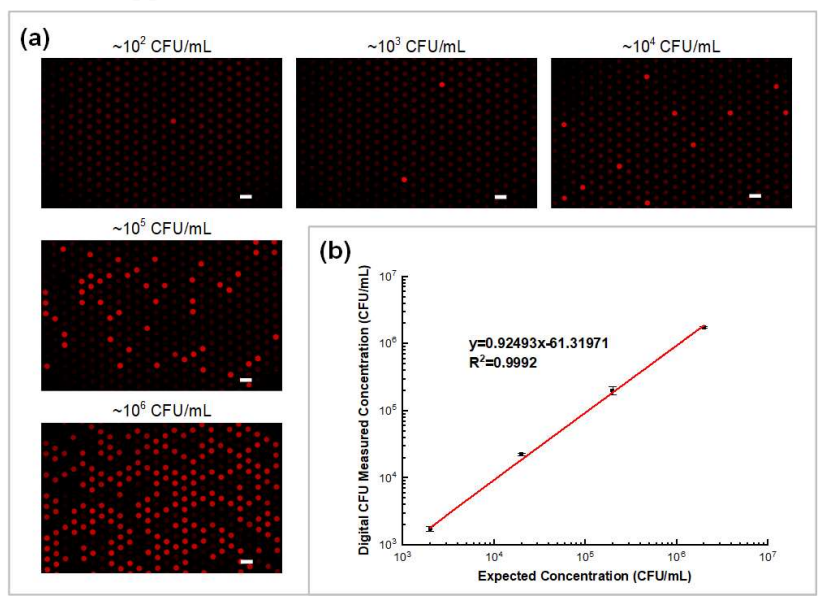

Figure 5. Digital enumeration of bacteria via the NMA chip. (a) Fluorescence images showing the representative results of growth of different concentration fluorescent E. coli JM109 in the NMA chip. Scale bar represents $200 \mu \mathrm{m}$. (b) A log plot showing the linear relationship between the measured concentrations with digital CFU assay and the expected concentration of E. coli JM109 samples whose concentrations ranged over four orders of magnitude (Note: each data point represents the mean \pm standard deviation of three independent experiments). Strong linearity between the calculated number of cells and the expected number of cells for E. coli JM109 indicates that the NMA chip can be applied for the precise enumeration of bacteria in a wide dynamic range.

Facile Fabrication of Highly Uniform Spherical Colloidal Photonic Crystals. Spherical colloidal photonic crystals (SCPCs), combining a large number of highly ordered nanoparticles in spherical geometry, possess unique photonic properties for various applications, such as optoelectronic devices, actuators, filters, sensors, and displays. ${ }^{35,36}$ To produce SCPCs, a variety of methods have been proposed. Among them, the droplet-template method is one of the most popular methods for the fabrication of SCPCs, in which droplets of aqueous colloidal suspension are dispensed on a hydrophobic surface or in oil and then dried to spontaneously assemble particles into close-packed spherical structures. ${ }^{37}$ To demonstrate the versatility of our self-digitization method, we further applied it to the fabrication of highly uniform SCPCs. Similar to digital PCR and digital CFU assays (more details see Figure S1), the first step was to discretize the sample into microwells. In this case, polystyrene (PS) suspension (235 nm in diameter) was used as the aqueous sample and n-hexadecane was used as the oil phase. Hexadecane has good wetting to PDMS, which allows hexadecane to penetrate into the interface between the aqueous phase and PDMS, resulting in the detachment of the confined aqueous sample from the microwell surface. Subsequently, due to the evaporation-induced shrinkage and energy minimization, the detached aqueous sample becomes a spherical droplet due to energy minimization. After all aqueous samples confined in microwells evolved into spherical shapes, the chips were placed in a convection oven for evaporating the aqueous solvent. The evaporation of the liquid medium leads to the assembly of PS nanoparticles into close-packed supraballs, i.e., the desired SCPCs. The size of the SCPCs is determined by the concentration of suspended colloidal particles. Figure 6 shows the SCPCs that were synthesized using our selfdigitization method. One of the main advantages of this method is its ability to synthesize highly monodispersed SCPCs. The statistical analysis indicates the synthesized SCPCs have a very narrow size distribution with a coefficient of variance $(\mathrm{CV})$ of $\sim 3.3 \%$ (Figure 6c), exhibiting an excellent monodispersion. It can also be found that they have a very regular hexagonal ordering of the nanoparticles at the surface (Figure 6b), which form the (111) lattice planes of a face centered-cubic (FCC) structure. Upon illumination by visible light, a strong green spot can be observed at the surface of each colloidal crystal sphere, which corresponds to the characteristic color of diffraction patterns. ${ }^{38}$ To further investigate the optical properties of the obtained SCPCs, we measured their reflection spectra by illuminating them with normal incidence light. As shown in Figure 6d, distinct peaks of reflection were observed at 563.6 $\mathrm{nm}$ for the SCPCs in the air, which correspond to the Bragg reflection generated mainly from the (111) planes of the SCPCs. According to Bragg's law, the value of the diameter of the particles assembling the SCPCs calculated from the results of the reflection spectrum is $236 \mathrm{~nm}$, which is in good agreement with the diameter of the PS nanoparticles used. In contrast to conventional droplet-template methods, this self-digitization method provides simple and robust fabrication of highly uniform SCPCs without the need for precise control of fluid, complex ink-jetting setups, or large volume sample, and thus has great potential for novel material synthesis in a wide variety of applications such as photonics, liquid crystals, and sensors.

\section{CONCLUSIONS}

In this work, we have developed a facile and rapid method for the discretization of samples using oil-triggered dewetting in a networked microwell array. Due to the large difference in Laplace pressure between the channel and well regions, the aqueous liquid primed in the networked microwell array is spontaneously isolated into microwells, achieving sample digitization without using any additional pumping or printing equipment. We demonstrated that this self-digitization method could be used for absolute quantitation of nucleic acids, digital enumeration of viable bacteria as well as facile synthesis of 
spherical particles, confirming its functionality and versatility. We believe that this simple, robust, and cost-effective sample digitization approach will find numerous applications for biological, medical, and synthetic purposes.

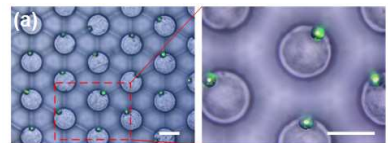

(c)
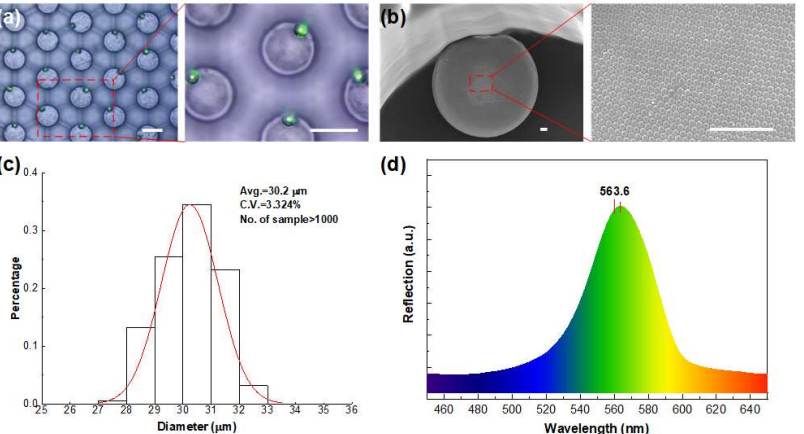

Figure 6. Preparation of spherical colloidal photonic crystals with the NMA chip. (a) Optical microscopy images of the spherical colloidal photonic crystals composed of PS nanoparticles with an average diameter of $235 \mathrm{~nm}$, scale bar is $100 \mu \mathrm{m}$. (b) SEM images of the spherical colloidal photonic crystals produced with the NMA chip, scale bar is $2 \mu \mathrm{m}$. (c) The statistical analysis for monodispersity of spherical colloidal photonic crystals produced with the NMA chip. The sizes of particles were measured by ImageJ software. (d) Normal reflection spectrum of the spherical colloidal photonic crystals consisting of PS nanoparticles with an average diameter of $235 \mathrm{~nm}$.

\section{EXPERIMENTAL SECTION}

Device Fabrication. The NMA chips were fabricated using standard soft lithography as described previously. ${ }^{39}$ Briefly, a multi-layer SU-8 procedure was performed to create two-layer patterns with SU-8 3010 and 3050 negative photoresists on a 4inch silicon wafer. Next, PDMS casting was performed to replicate PDMS microstructures from the SU-8 mold. According to different applications, PDMS was prepared with two different recipes. For the fabrication of the NMA chips used in digital microbial assays and synthesis of SCPCs, a standard recipe recommended by the supplier was used, in which PDMS monomer and cross-linker (Sylgard 184, Dow Corning, Midland, MI) were mixed in 10:1 (w/w); for the fabrication of the NMA chips used in digital PCR, a modified recipe from our previous experience was adopted, in which PDMS monomer, cross-linker and Triton X-100 (Sigma-Aldrich China, Shanghai, China) were mixed in 10:1:0.05 (w/w/w). In the latter PDMS recipe, the purpose of the addition of Triton X-100 is to modify the PDMS surface and then suppress the adsorption of DNA or enzymes to PDMS surfaces, which can significantly enhance PCR compatibility of the chip. ${ }^{24}$ After degassing for $1 \mathrm{~h}$, the freshly PDMS mixtures were poured over the molds and blank silicon wafer to create a microstructured PDMS layer and a flat PDMS cover layer, respectively. Subsequently, the casted PDMS was placed on a hotplate $\left(70^{\circ} \mathrm{C}\right)$ for $3 \mathrm{~h}$. After curing, the microstructured PDMS replica and the blank PDMS slab were peeled off from the mold and blank silicon wafer, respectively. Then, an inlet port and an oil reservoir were created on the PDMS cover layer with a punching tool and a scalpel, respectively. Next, the unstructured surface of the microstructured PDMS replica was bonded to a glass slide by plasma treatment. Finally, the PDMS cover layer and microstructured PDMS layer were reversibly assembled by alignment and conformal contact.
Numerical Simulation. The numerical simulation in this work was carried out using the COMSOL Multiphysics (v 5.6, Burlington, MA). For the simulations, we used the two connected microwells geometry (including the oil region above it) as the computational domain, and the numerical simulation was implemented using the Laminar Flow Two-Phase Flow, Level Set interface. Flow is assumed to be laminar, incompressible, Newtonian, and isothermal. The initial velocities of all liquid in the computational domain were set as 0 . All walls of microwells and microchannel were set as wetted walls. The oil/water/PDMS three-phase contact angle was determined by the sessile drop method combined with the Owens-Wendt equation and Young-Laplace equation (see Section S2 in Supporting Information for more details). All simulations were calculated with the same dimensions as those of microwells and microchannels in the prepared NMA chip (shown in Figure 1). All the materials and physical parameters used for simulations are detailed in the Supporting Information (Table S1).

PCR Reagents and Conditions. Genomic DNA containing KRAS G12A mutation was extracted from the A-549 lung carcinoma cell line using an animal tissue/cell genomic DNA extraction kit (Solarbio, Beijing, China). The extracted genomic DNA was quantified using NanoDrop spectrometer (ThermoFisher Scientific, Massachusetts, USA), then further diluted and realiquoted to use as templates in digital PCR. Here, Tris-EDTA buffer was used to dilute the extracted DNA to obtain a 10-fold serial dilution. The primers and probes used were chemically synthesized by Sangon Biotech Co., Ltd. (Shanghai, China). The sequences are given as follows (all in $5^{\prime} \rightarrow 3^{\prime}$ direction): forward primer, GCCTGCTGAAAATGACTGAATATAAACT; reverse primer, GCTGTATCGTCAAGGCACTCTT; probe, FAM-TTGGAGCTGCTGGCGTAMGB. The reaction volume of each digital PCR was $35 \mu \mathrm{L}$, containing $17.5 \mu \mathrm{L}$ of Premix Ex Taq (Probe qPCR) (Takara Bio, China), $1.8 \mu \mathrm{L}$ forward primer $(10 \mathrm{~nm}), 1.8 \mu \mathrm{L}$ reverse primer $(10 \mathrm{~nm}), 0.7 \mu \mathrm{L}$ probe $(10 \mathrm{~nm}), 1.7 \mu \mathrm{L}$ BSA (Takara Bio, China) $(10 \mathrm{mg} / \mathrm{mL}), 8 \mu \mathrm{L}$ RNase-free water and $3.5 \mu \mathrm{L}$ target template diluted by Tris-EDTA buffer. After sample discretization, the chips were transferred to a flat PCR system (MasterCycler Nexus flat, Eppendorf) to perform an amplification reaction. The thermal cycling protocol is given as follows: $95{ }^{\circ} \mathrm{C}$ hot starts for 30 seconds, 40 thermal cycles, $95{ }^{\circ} \mathrm{C}$ for 5 seconds, and $60^{\circ} \mathrm{C}$ for 34 seconds. In each digital PCR run, sterile double-distilled water was used as a negative control. All digital PCR experiments were performed in triplicate.

Bacterial Strains and Culture. The bacterial strain of E. coli JM109 was cultivated in Luria Bertani (LB) broth (Land Bridge, Beijing, China) at $37^{\circ} \mathrm{C}$ with a shaking rate of $220 \mathrm{rpm}$ overnight to obtain bacteria stock with cell density up to OD600 of 1.1. Then, a conventional plate-counting was carried out to measure the concentration of E. coli JM109 stock (see Section S3 and Figure S2 in Supporting Information for more details). For digital CFU detection, the E. coli stock was diluted with LB broth to obtain a serial dilution with concentrations ranging from $10^{3}$ to $10^{7}$ cells $/ \mathrm{mL}$. Subsequently, the diluted $E$. coli samples were mixed with AlamarBlue (Maokang Biotechnology Co., Ltd., Shanghai, China). Each final sample contained $90 \%$ dilution from $E$. coli stock and $10 \%$ AlamarBlue. In all experiments, the bacterial samples were immediately used after preparation. Next, the prepared bacterial samples were loaded in five pre-degassed NMA chips respectively for 
discretization. After discretization, the chips were incubated at $37^{\circ} \mathrm{C}$ for $4 \mathrm{~h}$ in an incubator (LHP-160, Jiebosen, Changzhou, China). Finally, the chips were taken out from the incubator and microscopically examined. For plate counting, $100 \mu \mathrm{L}$ from each of the diluted E. coli samples was plated in an agar plate. All agar plates were incubated at $37{ }^{\circ} \mathrm{C}$ for $16 \mathrm{~h}$. After incubation, the number of colonies on each plate was manually counted. Each measurement was performed in triplicate.

Preparation of SCPCs. The SCPCs were prepared using the microdroplet template method. The colloidal suspension containing $2.5 \mathrm{wt} \%$ PS nanospheres $(235 \mathrm{~nm}$ in diameter, Nanorainbow Biotechnology Co. Ltd, Nanjing, China) and nhexadecane (Aladdin, Shanghai, China) were used as the aqueous and oil phases, respectively. After discretization, the chip was immersed in a petri dish containing n-hexadecane. Next, the petri dish was placed in a $60{ }^{\circ} \mathrm{C}$ convection oven for $12 \mathrm{~h}$, allowing the aqueous solvent to be slowly evaporated. After evaporation-induced colloidal assembly, isopropanol (Aladdin, Shanghai, China) was used to remove excess hexadecane to obtain bare SCPCs. Finally, to enhance their mechanical strength, the bare SCPCs were thermally sintered at $110^{\circ} \mathrm{C}$ for $15 \mathrm{~min}$. The obtained SCPCs were analyzed using an optical microscope (SOPTOP CX40M) equipped with a CCD camera (touptek I3ISPM03100KPA, Hangzhou, China) and a scanning electron microscope (Zeiss Sigma 500, Carl Zeiss, Germany), respectively. In addition, microreflectance spectroscopy was performed using an inverted metallurgical microscope (SOPTOP ICX41M) coupled with an optic fiber spectrometer (Ocean Optics, USB2000+).

\section{ASSOCIATED CONTENT}

\section{Supporting Information}

The Supporting Information is available free of charge on the ACS Publications website.

Calculation of sample utilization rate during self-digitization with the NMA chip, measurement of oil/water/PDMS three-phase contact angle, and measurement of E. coli JM109 stock concentrations via plating; Figures including schematic diagrams for the fabrication procedure of spherical colloidal photonic crystals using our self-digitization method and measurement of E. coli JM109 stock concentration via plating; Table including the materials and physical parameters used for the numerical simulation (PDF)

Demonstration of the operation of the NMA chip for sample digitization (AVI)

Demonstration of the dynamic behavior of the oil-triggered, templated dewetting in a NMA chip (bird's eye view) (AVI)

Demonstration of the deformation/evoluation of oil/water interfaces after introducing an oil phase on the surface of a NMA chip (Cross-sectional view) (AVI)

Demonstration of the dynamic pressure mapping of the two-phase system in a NMA chip during the self-digitization process (Front view) (AVI)

\section{AUTHOR INFORMATION}

\section{Corresponding Author}

Qiang Zhao - Key Laboratory of Optoelectronic Technology and Systems, Ministry of Education, Chongqing University, Chongqing 400044, China; Email: qiangzh@cqu.edu.cn.
Gang Li - Key Laboratory of Optoelectronic Technology and Systems, Ministry of Education, Chongqing University, Chongqing 400044, China; E-mail: gang_li@cqu.edu.cn.

\section{Author Contributions}

The manuscript was written through contributions of all authors. / All authors have given approval to the final version of the manuscript.

Notes

The authors declare no competing financial interest.

\section{ACKNOWLEDGMENT}

This work was supported by the National Natural Science Foundation of China (No. 61974012, 21827812, and 52005063), the Natural Science Foundation of Chongqing (No. cstc2020jcyjmsxmX0188), and the National Postdoctoral Program for Innovative Talents (No. BX20190049).

\section{REFERENCES}

(1) Witters, D.; Sun, B.; Begolo, S.; Rodriguez-Manzano, J.; Robles, W.; Ismagilov, R. F., Digital biology and chemistry. Lab Chip 2014, 14 (17), 3225-3232.

(2) Basu, A. S., Digital assays part I: partitioning statistics and digital PCR. SLAS Technol. 2017, 22 (4), 369-386.

(3) Basu, A. S., Digital assays part II: digital protein and cell assays. SLAS Technol. 2017, 22 (4), 387-405.

(4) Zhang, Y.; Noji, H., Digital Bioassays: Theory, Applications, and Perspectives. Anal. Chem. 2017, 89 (1), 92-101.

(5) Rondelez, Y.; Tresset, G.; Tabata, K. V.; Arata, H.; Fujita, H.; Takeuchi, S.; Noji, H., Microfabricated arrays of femtoliter chambers allow single molecule enzymology. Nat. Biotechnol. 2005, 23 (3), 361365.

(6) Hindson, B. J.; Ness, K. D.; Masquelier, D. A.; Belgrader, P.; Heredia, N. J.; Makarewicz, A. J.; Bright, I. J.; Lucero, M. Y.; Hiddessen, A. L.; Legler, T. C.; Kitano, T. K.; Hodel, M. R.; Petersen, J. F.; Wyatt, P. W.; Steenblock, E. R.; Shah, P. H.; Bousse, L. J.; Troup, C. B.; Mellen, J. C.; Wittmann, D. K.; Erndt, N. G.; Cauley, T. H.; Koehler, R. T.; So, A. P.; Dube, S.; Rose, K. A.; Montesclaros, L.; Wang, S.; Stumbo, D. P.; Hodges, S. P.; Romine, S.; Milanovich, F. P.; White, H. E.; Regan, J. F.; Karlin-Neumann, G. A.; Hindson, C. M.; Saxonov, S.; Colston, B. W., High-Throughput Droplet Digital PCR System for Absolute Quantitation of DNA Copy Number. Anal. Chem. 2011, 83 (22), 8604-8610.

(7) Joensson, H. N.; Andersson Svahn, H., Droplet microfluidicsA tool for single - cell analysis. Angew. Chem. Int. Ed. 2012, 51 (49), 12176-12192.

(8) Hümmer, D.; Kurth, F.; Naredi-Rainer, N.; Dittrich, P. S., Single cells in confined volumes: microchambers and microdroplets. Lab Chip 2016, 16 (3), 447-458.

(9) Zheng, B.; Roach, L. S.; Ismagilov, R. F., Screening of protein crystallization conditions on a microfluidic chip using nanoliter-size droplets. J. Am. Chem. Soc. 2003, 125 (37), 11170-11171.

(10) Li, L.; Ismagilov, R. F., Protein crystallization using microfluidic technologies based on valves, droplets, and SlipChip. Annu. Rev Biophys. 2010, 39, 139-158

(11) Du, G.-S.; Pan, J.-Z.; Zhao, S.-P.; Zhu, Y.; den Toonder, J. M.; Fang, Q., Cell-based drug combination screening with a microfluidic droplet array system. Anal. Chem. 2013, 85 (14), 6740-6747.

(12) Courtney, M.; Chen, X.; Chan, S.; Mohamed, T.; Rao, P. P.; Ren, C. L., Droplet microfluidic system with on-demand trapping and releasing of droplet for drug screening applications. Anal. Chem. 2017, 89 (1), 910-915.

(13) McCallion, O. N.; Taylor, K. M.; Thomas, M.; Taylor, A. J., Nebulization of fluids of different physicochemical properties with airjet and ultrasonic nebulizers. Pharm. Res. 1995, 12 (11), 1682-1688.

(14) Diehl, F.; Li, M.; He, Y.; Kinzler, K. W.; Vogelstein, B.; Dressman, D., BEAMing: single-molecule PCR on microparticles in waterin-oil emulsions. Nat. Methods 2006, 3 (7), 551-559. 
(15) Teh, S. Y.; Lin, R.; Hung, L. H.; Lee, A. P., Droplet microfluidics. Lab Chip 2008, 8 (2), 198-220.

(16) Guo, M. T.; Rotem, A.; Heyman, J. A.; Weitz, D. A., Droplet microfluidics for high-throughput biological assays. Lab Chip 2012, 12 (12), 2146-2155.

(17) Baret, J.-C., Surfactants in droplet-based microfluidics. Lab Chip 2012, 12 (3), 422-433.

(18) Ottesen, E. A.; Hong, J. W.; Quake, S. R.; Leadbetter, J. R., Microfluidic Digital PCR Enables Multigene Analysis of Individual Environmental Bacteria. Science 2006, 314 (5804), 1464-1467.

(19) Shen, F.; Du, W.; Kreutz, J. E.; Fok, A.; Ismagilov, R. F., Digital PCR on a SlipChip. Lab Chip 2010, 10 (20), 2666-2672.

(20) Morrison, T.; Hurley, J.; Garcia, J.; Yoder, K.; Katz, A.; Roberts, D.; Cho, J.; Kanigan, T.; Ilyin, S. E.; Horowitz, D., Nanoliter high throughput quantitative PCR. Nucleic Acids Res. 2006, 34 (18), e123e123.

(21) Lin, B.; Guo, Z.; Geng, Z.; Jakaratanopas, S.; Han, B.; Liu, P., A scalable microfluidic chamber array for sample-loss-free and bubbleproof sample compartmentalization by simple pipetting. Lab Chip 2020, 20 (16), 2981-2989.

(22) Feng, W.; Ueda, E.; Levkin, P. A., Droplet Microarrays: From Surface Patterning to High-Throughput Applications. Adv. Mater. 2018, 30 (20), e1706111.

(23) Zhu, Q.; Gao, Y.; Yu, B.; Ren, H.; Qiu, L.; Han, S.; Jin, W.; Jin, Q.; Mu, Y., Self-priming compartmentalization digital LAMP for point-of-care. Lab Chip 2012, 12 (22), 4755-4763.

(24) Fu, Y.; Zhou, H.; Jia, C.; Jing, F.; Jin, Q.; Zhao, J.; Li, G., A microfluidic chip based on surfactant-doped polydimethylsiloxane (PDMS) in a sandwich configuration for low-cost and robust digital PCR. Sensors Actuators B: Chem. 2017, 245, 414-422.

(25) Ning, Y.; Cui, X.; Yang, C.; Jing, F.; Bian, X.; Yi, L.; Li, G., A self-digitization chip integrated with hydration layer for low-cost and robust digital PCR. Anal. Chim. Acta 2019, 1055, 65-73.

(26) Cui, X.; Wu, L.; Wu, Y.; Zhang, J.; Zhao, Q.; Jing, F.; Yi, L.; Li, G., Fast and Robust Sample Self-Digitization for Digital PCR. Anal. Chim. Acta 2020, 1107, 127-134.

(27) Cui, X.; Hu, T.; Chen, Q.; Zhao, Q.; Wu, Y.; Xie, T.; Liu, P.; $\mathrm{Su}, \mathrm{X}$.; Li, G., A facile and rapid route to self-digitization of samples into a high density microwell array for digital bioassays. Talanta 2021, $233,122589$.

(28) Hosokawa, K.; Sato, K.; Ichikawa, N.; Maeda, M., Power-free poly (dimethylsiloxane) microfluidic devices for gold nanoparticlebased DNA analysis. Lab Chip 2004, 4 (3), 181-185.
(29) Li, G.; Luo, Y.; Chen, Q.; Liao, L.; Zhao, J., A "place n play" modular pump for portable microfluidic applications. Biomicrofluidics 2012, $6(1), 014118$

(30) Jackman, R. J.; Duffy, D. C.; Ostuni, E.; Willmore, N. D.; Whitesides, G. M., Fabricating large arrays of microwells with arbitrary dimensions and filling them using discontinuous dewetting. Anal. Chem. 1998, 70 (11), 2280-2287.

(31) Kim, H. U.; Roh, Y. H.; Mun, S. J.; Bong, K. W., Discontinuous Dewetting in a Degassed Mold for Fabrication of Homogeneous Polymeric Microparticles. ACS Appl. Mater. Inter. 2020, 12 (47), 53318 53327

(32) Altomare, M.; Nguyen, N. T.; Schmuki, P., Templated dewetting: designing entirely self-organized platforms for photocatalysis. Chem. Sci. 2016, 7 (12), 6865-6886.

(33) Peeters, M.; Douillard, J.-Y.; Van Cutsem, E.; Siena, S.; Zhang, K.; Williams, R.; Wiezorek, J., Mutant KRAS codon 12 and 13 alleles in patients with metastatic colorectal cancer: assessment as prognostic and predictive biomarkers of response to panitumumab. J. Clin. Oncol. 2013, 31 (6), 759-765.

(34) Hsieh, K.; Zec, H. C.; Chen, L.; Kaushik, A. M.; Mach, K. E.; Liao, J. C.; Wang, T.-H., Simple and precise counting of viable bacteria by resazurin-amplified picoarray detection. Anal. Chem. 2018, 90 (15), 9449-9456.

(35) Zhao, Y.; Shang, L.; Cheng, Y.; Gu, Z., Spherical colloidal photonic crystals. Acc. Chem. Res. 2014, 47 (12), 3632-3642.

(36) Wang, H.; Zhao, Z.; Liu, Y.; Shao, C.; Bian, F.; Zhao, Y., Biomimetic enzyme cascade reaction system in microfluidic electrospray microcapsules. Sci. Adv. 2018, 4 (6), eaat 2816.

(37) Cho, Y.-S.; Yi, G.-R.; Kim, S.-H.; Pine, D. J.; Yang, S.-M., Colloidal clusters of microspheres from water-in-oil emulsions. Chem Mater. 2005, 17 (20), 5006-5013.

(38) Vogel, N.; Utech, S.; England, G. T.; Shirman, T.; Phillips, K. R.; Koay, N.; Burgess, I. B.; Kolle, M.; Weitz, D. A.; Aizenberg, J., Color from hierarchy: Diverse optical properties of micron-sized spherical colloidal assemblies. Proc. Natl. Acad. Sci. USA 2015, 112 (35), $10845-10850$

(39) Mata, A.; Fleischman, A. J.; Roy, S., Fabrication of multi-layer SU-8 microstructures. J. Micromech. Microeng. 2006, 16 (2), 276-284.

For TOC only

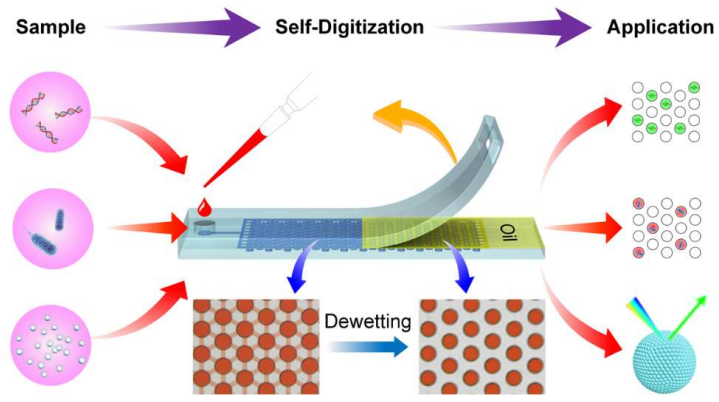

